Portland State University

PDXScholar

Electrical and Computer Engineering Faculty

Publications and Presentations

$4-1-1974$

\title{
Output Properties of Short-Pulse Gain-Switched Lasers
}

Lee W. Casperson

Portland State University

Follow this and additional works at: https://pdxscholar.library.pdx.edu/ece_fac

Part of the Electrical and Computer Engineering Commons

Let us know how access to this document benefits you.

\section{Citation Details}

Lee W. Casperson, "Output Properties of Short-Pulse Gain-Switched Lasers," Appl. Opt. 14, 299-305 (1975).

This Article is brought to you for free and open access. It has been accepted for inclusion in Electrical and Computer Engineering Faculty Publications and Presentations by an authorized administrator of PDXScholar. Please contact us if we can make this document more accessible: pdxscholar@pdx.edu. 


\title{
Output Properties of Short-Pulse Gain-Switched Lasers
}

\author{
Lee W. Casperson
}

\begin{abstract}
A formalism is derived for analyzing the spectral and temporal properties of gain-switched lasers. The development of the longitudinal mode structure from spontaneous emission, limiting linewidths, and over-all spectral characteristics are considered in detail; and applications are discussed.
\end{abstract}

\section{Introduction}

In the recent past, substantial research effort has been devoted to the development of lasers operating in the $u v$ and $x$-ray regions of the spectrum. Because of the unfavorable ratio of spontaneous to stimulated emission for these wavelengths, most of the current and proposed lasers operate on a short-pulse basis. Other lasers such as the $\mathrm{CO}_{2}$ TEA devices are pulsed to enhance the peak output power. Previous treatments of gain-switching and $Q$-switching have considered a single monochromatic radiation field at line center in a homogeneously broadened medium. ${ }^{1}$ In gas lasers, however, Doppler broadening is not necessarily negligible. Short-pulse lasers also typically operate with a large number of closely spaced longitudinal modes, and the single mode approximation is not valid. We present here a formalism governing the development of the output laser pulse from the initial broadband spontaneous emission including the formation and resolution of the modes. The results can be applied in a straightforward way to the interpretation or prediction of the output properties of pulsed lasers.

In Sec. II is a detailed derivation of the development of the longitudinal mode structure from spontaneous emission. It is found that under most conditions the spectral width of the modes rapidly narrows to a limiting value that may remain nearly constant throughout the rest of the pulse. This width is generally much greater than the linewidths that are familiar for typical $\mathrm{cw}$ laser oscillators. In very shortpulse systems, the transient nature of the fields and of the excitation exclude altogether the development of discrete longitudinal modes. In Sec. III is derived the noise input to the laser in terms of fundamental

The author is with the University of California, School of Engineering and Applied Science, Los Angeles, California 90024.

Received 24 April 1974. properties of the amplifying medium. The resultant linewidth formulas are specialized to the case of $\mathrm{cw}$ oscillation for comparison with previous results. It is shown in Sec. IV that the over-all spectral envelope also narrows with time to a width that may be much less than the initial spontaneous emission linewidth. Discussions are included in Sec. V regarding the applicability of these results to practical short-pulse lasers.

\section{Formation of The Longitudinal Modes}

In this section the longitudinal mode structure of a short-pulse laser oscillator is derived. The model used is that of a plane parallel Fabry-Perot interferometer containing an amplifying laser medium. The gain of the medium is switched on instantaneously at time $t=0$, as is a broadband spontaneous emission source within the cavity. With increasing time the radiation is amplified as it makes increasing numbers of passes through the cavity. The superposition of fields that are initiated by spontaneous emission at various points in time leads to the longitudinal mode spectrum of the laser. This instantaneous switching model is a reasonable approximation for many modern laser systems where transverse pumping or high speed shuttering yield turn-on times comparable with the cavity round trip time.

One set of fields in the resonator is shown schematically in Fig. 1. The amplitude of the spontaneously emitted radiation traveling to the right at a point near the right-hand end of the resonator is $E_{1}$. This is light that has made exactly one round trip. The small arrows indicate the reduction of the amplitude due to the mirror amplitude reflectivities $r_{l}$ and $r_{r}$. Then the radiation with amplitude $E_{1}$ will after $n-1$ additional passes have the amplitude

$$
E_{n}=E_{1}\left(r_{l} r_{r} \exp -i \oint k d z\right)^{n-1}
$$

where $k=\beta+i \alpha$ is the complex propagation constant and the integral represents one loop around. the cavity. Initially it is assumed that $k$ is independent 


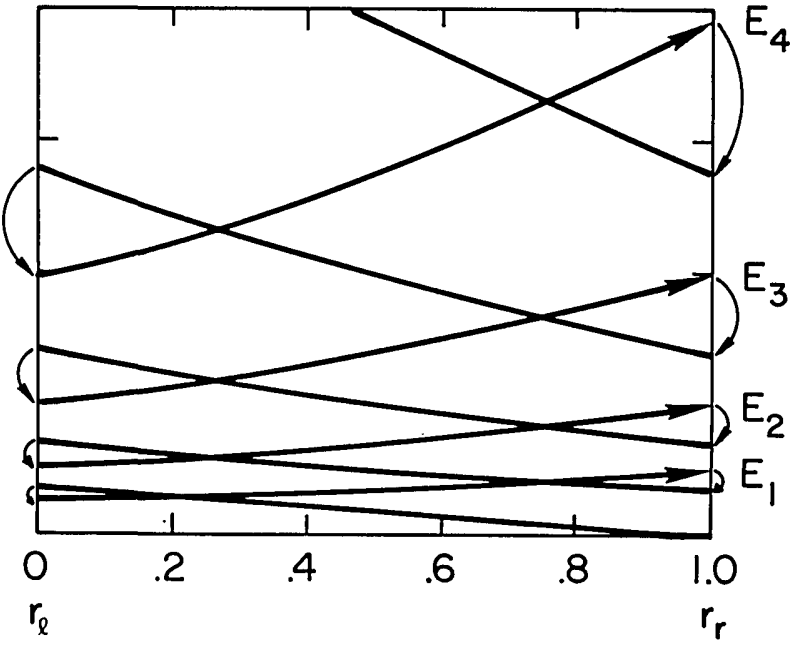

Fig. 1. Development of the electromagnetic fields from spontaneous emission in an active Fabry-Perot laser resonator. Mirror reflectivities are $r_{l}=r_{r}=0.7$, and the amplitude gain constant is 0.7 $l^{-1}$, where $l$ is the resonator length.

of time and frequency, and saturation effects are considered later.

By the time the original radiation has made $n$ passes through the cavity, the total electric field $E_{t}$ will be the sum of $E_{n}$ and the radiation that originated at a later time or

$$
\begin{aligned}
E_{t}=E_{n}\left(1+E_{1} / E_{2}+E_{1} / E_{3}+\ldots+E_{1} / E_{n}\right) \\
=E_{1}\left(r_{l} r_{r} \exp -i \oint k d z\right)^{n-1}\left[1+\left(r_{l} r_{r} \exp -i \oint k d z\right)^{-1}\right. \\
+\left(r_{l} r_{r} \exp -i \oint k d z\right)^{-2} \\
\left.+\cdots+\left(r_{l} r_{r} \exp -i \oint k d z\right)^{-(n-1)}\right] .
\end{aligned}
$$

This is a geometrical series having the sum

$$
\begin{aligned}
E_{t}=E_{1}\left\{\left[1-\left(r_{l} r_{r} \exp -i \oint k d z\right)^{n}\right] /\right. & {\left[1-\left(r_{l} r_{r} \exp \right.\right.} \\
& -i \oint k d z)]\} .
\end{aligned}
$$

In terms of the intensity $I \alpha E E^{*}$, Eq. (3) becomes

$I_{t}=$

$I_{1}=\frac{\left[1-\left(r_{l} r_{r} \exp -i \oint k d z\right)^{n}\right]\left[1-\left(r_{l}{ }^{*} r_{r}{ }^{*} \exp i \oint k^{*} d z\right)^{n}\right]}{\left[1-\left(r_{l} r_{r} \exp -i \oint k d z\right)\right]\left[1-\left(r_{l}{ }^{*} r_{r}{ }^{*} \exp i \oint k^{*} d z\right)\right]}$

The amplitude reflectivities $r_{l}$ and $r_{r}$ are generally complex to account for phase changes on reflection. These phase changes depend on the mirror material and the choice of reference plane. We define the reflectivities in terms of the intensity reflection coefficients according to

$$
r_{l}=R_{l}{ }^{1 / 2} \exp -i \phi_{l} ; r_{r}=R_{r}{ }^{1 / 2} \exp -i \phi_{r} .
$$

Then Eq. (4) reduces to

$$
\begin{aligned}
I_{t}=I_{1} \frac{-2\left(R_{l} R_{r} \exp 2 \oint \alpha d z\right)^{n / 2} \cos n\left(\phi \beta d z+\phi_{l}+\phi_{r}\right.}{1+\left(R_{l} R_{r} \exp 2 \oint \alpha d z\right)} \\
-2\left(R_{l} R_{r} \exp 2 \oint \alpha d z\right)^{1 / 2} \cos \left(\oint \beta d z+\phi_{l}+\phi_{r}\right)
\end{aligned} .
$$

The real part of the propagation constant is related to the frequency $\nu$, the index of refraction $n_{o}$, and the vacuum speed of light by $\beta=2 \pi n_{o} \nu / c$. In terms of the normalized frequency $x=\nu \oint\left(2 \pi n_{o} / c\right) d z+\phi_{l}+$ $\phi_{r}$ and the effective single pass gain factor $F=R_{l}^{1 / 2}$ $R_{r}{ }^{1 / 2} \exp \phi \alpha d z$, Eq. (6) may be written

$$
\begin{array}{r}
I_{t}=I_{1}\left[\left(1+F^{2 n}-2 F^{n} \cos n x\right) /\left(1+F^{2}-2 F \cos x\right)\right] \\
=I_{1}\left\{\left[\left(F^{n}-1\right)^{2}+4 F^{n} \sin ^{2}(n x / 2)\right] /\left[(F-1)^{2}\right.\right. \\
\left.\left.\cdot+4 F \sin ^{2}(x / 2)\right]\right\} .
\end{array}
$$

Equation (7) describes the longitudinal mode spectrum of an unsaturated gain-switched laser in which the radiation has had time to make $n$ round trips through the cavity. The intensity peaks or longitudinal modes are at the frequencies $x_{j}=2 \pi j$, where $j$ is an integer. To see the implications of this result, Eq. (7) is plotted in Fig. 2 for a single pass gain of $F=1.5$. The broadband spontaneous emission spectrum is transformed after a few round trips into a resonant mode structure, and then no further narrowing of the resonance lines occurs. That the spectrum should become independent of $n$ follows from Eq. (7) because for $F^{n} \gg 1$ the intensity is governed by

$$
I_{t}=\left\{\left(I_{1} F^{2 \pi}\right) /\left[(F-1)^{2}+4 F \sin ^{2}(x / 2)\right]\right\} .
$$

The derivation of the noise input $I_{1}$ is given in Sec. III.

The condition $F^{n} \gg 1$ must eventually be satisfied in all practical gain-switched lasers if the output is to exceed the noise level. Accordingly, it is important to consider what the limiting mode structure is for various values of $F$; and a plot of Eq. (8) is given in Fig. 3. It is clear from this figure that if $F$ is significantly greater than unity, the resonance lines become

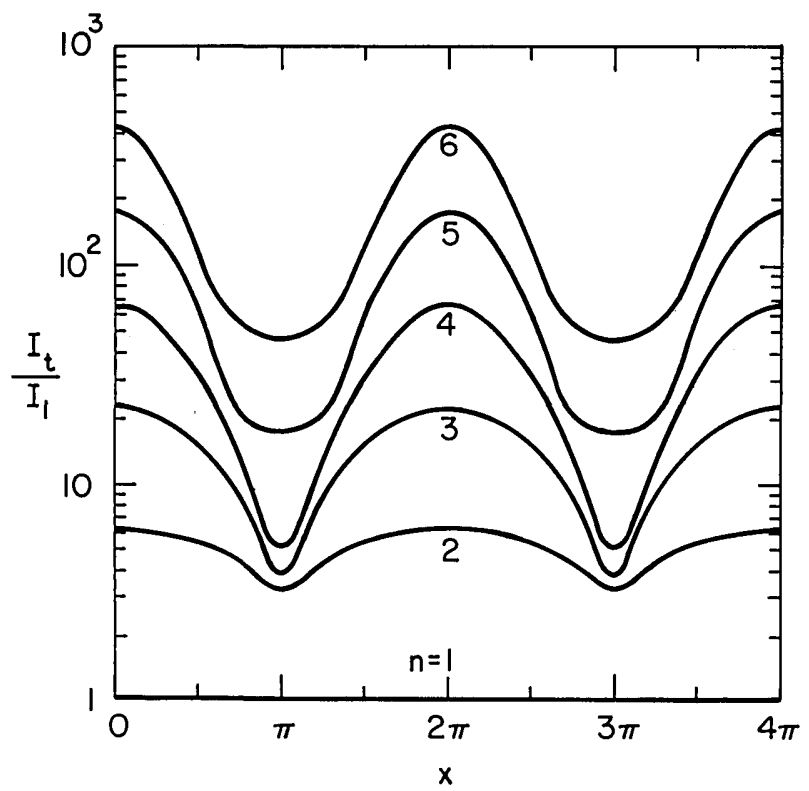

Fig. 2. Intensity spectrum incident on the right-hand mirror for increasing numbers $n$ of round trips through the cavity with a single-pass gain factor $F=1.5$. 


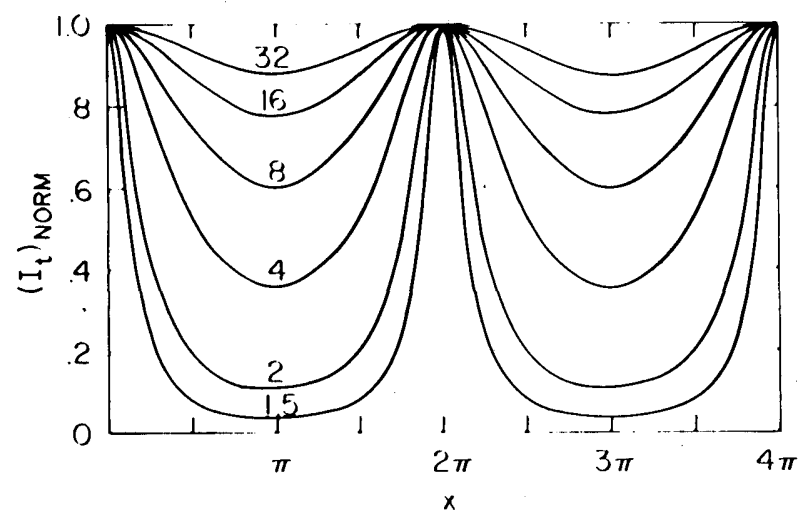

Fig. 3. Limiting emission spectra for various values of the singlepass gain factor $F>1$ with the emission maxima normalized to unity.

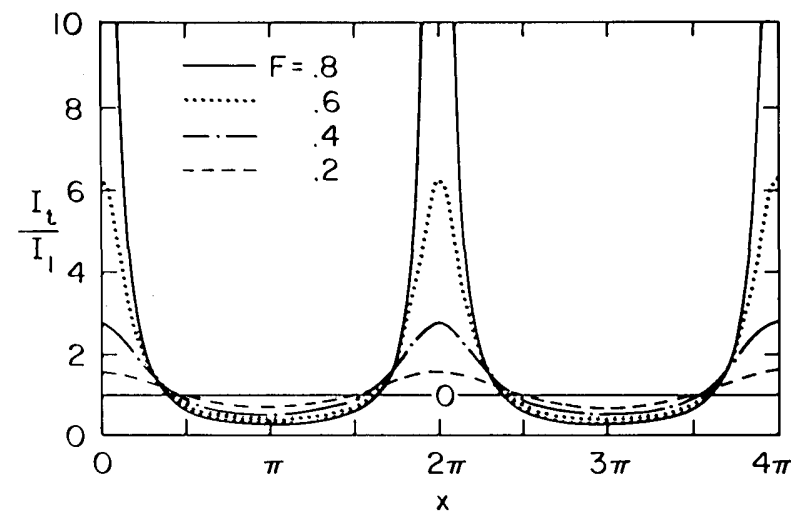

Fig. 4. Limiting emission spectra for various values of the singlepass gain factor $F<1$.

much wider than in typical cw lasers. For $F>10$, the mode structure begins to disappear altogether. One can readily show from Eq. (8) that when the limiting resonance lines are narrow compared with the mode spacing, the full width at half maximum is

$$
\Delta x_{\text {mode }}=2(F-1) / F^{1 / 2} \text {. }
$$

If the gain is less than the loss $(F<1)$, then from Eq. (7) the limiting mode structure is

$$
I_{t}=I_{1} /\left[(1-F)^{2}+4 F \sin ^{2}(x / 2)\right] .
$$

This result can also be derived from the requirement that the fields must repeat after one round trip according to $E_{t}-E_{1}=E_{t} r_{l} r_{r}$ exp-i $\varnothing k d z$. Equation (10) is plotted in Fig. 4 for various values of $F$. Evidently resonance lines exist even when the gain is less than the loss. Thus the development of a mode structure when a gain-switched medium is operated in a laser cavity is by itself not a sufficient test for stimulated emission. The linewidth for this case is the negative of the value given in Eq. (9). It is interesting also to note that this corresponds to the operation of steady-state laser oscillators. In oscillators the very slight discrepancy between the loss and the gain is made up by spontaneous emission added to the laser mode. It is shown in Sec. III that these results may be used to determine the steady-state oscillation linewidth.

Sometimes it is more important to know the total intensity associated with a particular laser mode than it is to know the details of the intensity spectrum. The mode intensity may be found from the integral

$$
I_{\text {mode }}=\int_{\nu_{j}-(\Delta \nu) / 2}^{\nu_{j}+(\Delta \nu) / 2} I_{t}(\nu) d \nu
$$

where $\nu_{j}=\left(2 \pi j-\phi_{l}-\phi_{r}\right) / \oint\left(2 \pi n_{o} / c\right) d z$ is the center frequency of the mode and $\Delta \nu=2 \pi / \oint\left(2 \pi n_{o} / c\right) d z$ is the intermode frequency spacing. With Eq. (7) the result is ${ }^{2}$

$$
\begin{aligned}
I_{\text {mode }} & =\left(\Delta \nu I_{1}\right) /(2 \pi) \int_{-\pi}^{\pi}\left[\left(1+F^{2 n}-2 F^{n} \cos n x\right) /\left(1+F^{2}\right.\right. \\
& =\Delta \nu I_{1}\left[\left(F^{2 n}-1\right) /\left(F^{2}-1\right)\right] .
\end{aligned}
$$

After many passes this reduces to

$$
I_{\text {mode }}= \begin{cases}\left(\Delta \nu I_{1} F^{2 n}\right) /\left(F^{2}-1\right) & F>1 \\ \left(\Delta \nu I_{1}\right) /\left(1-F^{2}\right) & F<1 .\end{cases}
$$

From these equations follows the practical result that the unsaturated output of the laser increases with increases in reflectivity $R_{r}$ of the coupling mirror if and only if the condition $R_{l} \exp (2 \oint \alpha d z)>1$ is satisfied.

It has been assumed here that the gain remains constant while the radiation makes an increasing number of passes through the cavity. However, if the radiation experiences a net gain as it propagates, it must eventually reach a level sufficient to saturate the amplifying medium. An examination of the significance of the terms in Eq. (2) shows that if saturation first occurs after $m$ round trips through the cavity, the $m$ th term in the summation is the first to be affected. But the summation is dominated by the leading terms, so the saturation does not affect the spectrum. Thus the longitudinal mode spectrum is determined primarily by the initial value of the gain and the input $I_{1}(\nu)$ (this could be an injected signal rather than spontaneous emission), and only the over-all intensity is influenced by the saturation.

\section{Effective Noise Input}

In the previous section, several relationships have been derived governing the output longitudinal mode structure of a gain-switched laser. All of these results depend, however, on knowing the effective noise intensity $I_{1}$ (or the injected signal), which is incident initially on the right-hand resonator mirror. The purpose of this section is to obtain explicit expressions for $I_{1}$ due to spontaneous emission noise in terms of more fundamental parameters of the laser system.

By the noise intensity $I_{1}$ we mean the noise that originated within the amplifying medium but has 
made less than a full round trip through the resonator. Thus there are two contributions to $I_{1}$. The first consists of radiation that is emitted toward the right-hand mirror and undergoes amplification as it propagates. The second consists of radiation that is emitted in the left-hand direction, is amplified by the medium, is reflected from the left-hand mirror, and is amplified again before arriving at the right-hand mirror. The paraxial noise per mode emitted from one end of a uniform mirrorless homogeneously broadened laser medium can be written ${ }^{3}$

$$
\begin{aligned}
N(\nu) d \nu & =\left(\left\{h c^{2} N_{2} L(\nu)[\exp (2 \alpha(\nu) l)-1] d v\right\} /\left[16 \pi \nu \alpha(\nu) t_{s}\right]\right) \\
& =\left[\left(h \nu N_{2}\right) /\left(N_{2}-N_{1}\right)\right][\exp (2 \alpha(\nu) l)-1] d \nu,
\end{aligned}
$$

where $N_{2}$ and $N_{1}$ are, respectively, the total population densities of the upper and lower laser levels, $c$ is the speed of light in the medium of length $l, t_{s}$ is the spontaneous emission lifetime, and $L(\nu)$ is the normalized line shape function. Equation (15) is valid also for inhomogeneous broadening before saturation or whenever the lower level population can be neglected. If we consider now the effect of the lefthand mirror, it is clear from Eq. (15) and the preceding discussion that the effective noise intensity incident on the right-hand mirror is

$$
\begin{array}{r}
I_{1}=\left\{\left(h \nu N_{2}\right) /\left[A_{r}\left(N_{2}-N_{1}\right)\right]\right\}\left[1+R_{l} \exp (2 \alpha l)\right][ \\
\exp (2 \alpha l) \\
-1],(16)
\end{array}
$$

where $A_{r}$ is the area of the beam at the right-hand mirror. Equation (16) is an expression for the input noise intensity to a gain-switched laser oscillator. Use of this equation in the results of Sec. II yields quantitative formulas for the development of the longitudinal modes.

This Fabry-Perot analysis can be compared with previous treatments by considering the special case of steady-state oscillation. Equations (13) and (16) imply that the total power in a single mode leaving the cavity through the right-hand mirror is (assuming lossless mirrors)

$$
\begin{aligned}
P_{r}=\left\{\left(h \nu N_{2} \Delta \nu\right) /\left[\left(N_{2}-N_{1}\right)\left(1-F^{2}\right)\right]\right\}[1 \\
\left.+R_{l} \exp (2 \alpha l)\right][\exp (2 \alpha l)-1]\left(1-R_{r}\right) .
\end{aligned}
$$

Adding to this, a similar expression for the left-hand mirror yields the total output power in the mode

$$
\begin{aligned}
P_{\text {mode }} & =\left\{\left(h \nu N_{2} \Delta \nu\right) /\left[\left(N_{2}-N_{1}\right)\left(1-F^{2}\right)\right]\right\}[\exp (2 \alpha l)-1]\{2 \\
& \left.-2 R_{l} R_{r} \exp (2 \alpha l)+\left(R_{l}+R_{r}\right)[\exp (2 \alpha l)-1]\right\} .
\end{aligned}
$$

Far above threshold $\exp (2 \alpha l) \simeq\left(R_{l} R_{r}\right)^{-1 / 2}$; and Eq. (18) reduces to

$$
\begin{aligned}
P_{\text {mode }} & \simeq \frac{h \nu N_{2} \Delta \nu\left[1-\left(R_{l} R_{r}\right)^{1 / 2}\right]^{2}\left(R_{l}{ }^{1 / 2}+R_{r}{ }^{1 / 2}\right)^{2}}{2\left(N_{2}-N_{1}\right)(1-F) R_{t} R_{r}} \\
& =\frac{h \nu N_{2}(\Delta \nu)^{2}\left[1-\left(R_{l} R_{r}\right)^{1 / 2}\right]^{2}\left(R_{l}{ }^{1 / 2}+R_{r}{ }^{1 / 2}\right)}{2 \pi\left(N_{2}-N_{1}\right) \Delta \nu_{\text {mode }} R_{l} R_{r}}, \text { (19) }
\end{aligned}
$$

where $F \simeq 1$, and Eq. (9) has been used to express the linewidth of the mode as

$$
\Delta \nu_{\text {mode }}=[\Delta \nu(1-F)] /\left(\pi F^{1 / 2}\right) .
$$

If the empty cavity linewidth $\Delta \nu_{c}=\Delta \nu\left[1-\left(R_{l} R_{r}\right)^{1 / 2}\right]$ $/\left[\pi\left(R_{l} R_{r}\right)^{1 / 4}\right]$ is introduced, Eq. (19) becomes

$$
\begin{aligned}
\Delta \nu_{\text {mode }}=\left\{\left[2 \pi h \nu N_{2}\left(\Delta \nu_{c}\right)^{2}\right] /[\right. & \left.\left.\left(N_{2}-N_{1}\right) P_{\text {mode }}\right]\right\}\left[\left(R_{l}{ }^{1 / 2}\right.\right. \\
& \left.\left.+R_{r}{ }^{1 / 2}\right) /\left(2 R_{l}{ }^{1 / 4} R_{r}{ }^{1 / 4}\right)\right]^{2} .
\end{aligned}
$$

This result has also been obtained from a transmission line analog, ${ }^{4}$ and it differs by the factor involving mirror reflectivities from the results of microwave cavity $^{5}$ and circuit ${ }^{6}$ models. The mirror factor could be significant for high loss asymmetric resonators operated near threshold. ${ }^{7}$ For example, with $R_{l}=1$ and $R_{r}=0.04$, the value of this factor is 1.8.

\section{Spectral Envelope}

The previous discussion has shown that in a gainswitched laser the spontaneous emission spectrum is gradually resolved into longitudinal modes. The rate and extent of this process depend on the single pass gain factor $F$. In addition the over-all spectral envelope may also be expected to evolve in time, because initially the gain is greater near line center than in the wings of the transition spectrum. It is found that before saturation the output linewidth may narrow to a value much less than the width of the original noise spectrum. The mode spacing (if discrete modes develop) is assumed to be much less than the homogeneous linewidth. This condition is satisfied in practical short-pulse lasers.

The starting point for this analysis is the pair of rate equations governing the frequency-dependent population densities in a medium with inhomogeneous broadening:

$$
\begin{aligned}
& \frac{\partial n_{2}(\nu, z, t)}{\partial t}= S_{2}(\nu, t)-n_{2}(\nu, z, t)\left[A_{2}\right. \\
&\left.+\frac{2 B_{o}}{\pi \Delta \nu_{h}} \int_{-\infty}^{-\infty} \frac{I\left(y_{n}, z, t\right) d y_{n}}{1+\left(y-y_{n}\right)^{2}}\right] \\
& \quad+n_{1}(\nu, z, t) \frac{2 B_{0}}{\pi \Delta \nu_{h}} \int_{-\infty}^{\infty} \frac{I\left(y_{n}, z, t\right) d y_{n}}{1+\left(y-y_{n}\right)^{2}} ; \\
& \frac{\partial n_{1}(\nu, z, t)}{\partial t}= S_{1}(\nu, t)+n_{2}(\nu, z, t)\left[A_{21}\right. \\
&\left.+\frac{2 B_{o}}{\pi \Delta \nu_{h}} \int_{-\infty}^{\infty} \frac{I\left(y_{n}, z, t\right) d y_{n}}{1+\left(y-y_{n}\right)^{2}}\right]-n_{1}(\nu, z, t)\left[A_{1}\right. \\
&\left.\quad+\frac{2 B_{o}}{\pi \Delta \nu_{h}} \int_{-\infty}^{\infty} \frac{I\left(y_{n}, z, t\right) d y_{n}}{1+\left(y-y_{n}\right)^{2}}\right] .
\end{aligned}
$$

These are identical to the equations of Gordon et al.,8 except that the interaction has been generalized to a broadband optical continuum rather than a single monochromatic mode, and an explicit Lorentzian frequency dependence of the Einstein $B$ coefficient has been introduced with a line center value of $2 B_{o} / \pi \Delta \nu_{h}$. $S_{1}(\nu, t)$ and $S_{2}(\nu, t)$ are time dependent pumping rates, $y_{n}=2\left(\nu_{n}-\nu_{0}\right) / \Delta \nu_{h}$ is a frequency parameter normalized in units of the homogeneous linewidth $\Delta \nu_{h}$, and $I\left(y_{n}, z, t\right)$ is the average spectral density at the frequency $y_{n}$. If the intensity were monochromatic, these equations then would reduce to those given 
previously. ${ }^{8}$ In the usual gain-switched situation, pumping and spontaneous relaxation of the upper level have a negligible effect on the population densities during the short laser pulse. Then if the lower state decays quickly compared with the pulse length (as in uv transitions to a repulsive lower state), it follows from Eqs. (22) and (23) that the inversion density $n=n_{2}-n_{1}$ is governed by

$$
\frac{\partial n(\nu, z, t)}{\partial t}=-n(\nu, z, t) \frac{2 B_{o}}{\pi \Delta \nu_{h}} \int_{-\infty}^{\infty} \frac{I\left(y_{n}, z, t\right) d y_{n}}{1+\left(y-y_{n}\right)^{2}} .
$$

(In the opposite limit of negligible lower state decay, the right-hand side of Eq. (24) would be multiplied by two.) The integral of this equation is

$$
n(\nu, z, t)=p(\nu) \exp \left[-\frac{2 B_{o}}{\pi \Delta \nu_{h}} \int_{0}^{t} \int_{-\infty}^{\infty} \frac{I\left(y_{n}, z, t^{\prime}\right) d y_{n} d t^{\dagger}}{1+\left(y-y_{n}\right)^{2}}\right]
$$

where $p(\nu)=p_{o} \exp \left(-\epsilon^{2} y^{2}\right)$ is the inversion spectrum before the onset of the saturating laser pulse and $\epsilon=$ $\left(\Delta \nu_{h} / \Delta \nu_{\mathrm{D}}\right)(1 n 2)^{1 / 2}$ measures the relative importance of homogeneous and Doppler broadening.

The gain is proportional to the population inversion of Eq. (25), and one can show that the time dependence of the intensity spectrum is governed by

$$
\begin{aligned}
\frac{\partial I\left(y_{t}, t\right)}{\partial t}= & \frac{k\left[I\left(y_{l}, t\right)+\eta\right]}{\pi} \int_{-\infty}^{\infty} \frac{\exp \left(-\epsilon^{2} y^{2}\right)}{1+\left(y-y_{l}\right)^{2}} \\
& \times \exp \left[-s \int_{0}^{t} \int_{-\infty}^{-\infty} \frac{I\left(y_{n}, t^{\prime}\right) d y_{n} d t^{\prime}}{1+\left(y-y_{n}\right)^{2}}\right] d y,
\end{aligned}
$$

where the gain parameter is $k=4 l h \nu_{l} B_{o} p_{o} /\left(\pi T \Delta \nu_{h}\right)$, with $l$ the length of the amplifying medium and $T$ the round trip time. The noise parameter is $\eta=$ $h \nu_{l} \Delta \nu_{h} /(2 A)$. The saturation parameter is $s=2 B_{o} /$ $\left(\pi \Delta \nu_{h}\right) ;$ and the loss parameter is $\gamma=(2 \delta l-$ $\left.1 n R_{l} R_{r}\right) / T$, where $\delta$ represents distributed losses in the laser medium due, for example, to photoionization in uv lasers. The intensity equation was written in this form to emphasize the close analogy between this result and the equation governing the spatial dependence of the intensity spectrum in a high gain laser amplifier. ${ }^{9}$ The only difference is that the spatial variable $z$ is replaced here by the time variable $t$, and the saturation factor has a different form.

In an unsaturated oscillator the exponential saturation factor in Eq. (26) reduces to unity, and the linewidth results for laser amplifiers can be adopted directly. For brevity, we only indicate a few of the most useful conclusions. In an unsaturated homogeneously broadened laser oscillator $(\epsilon \gg 1)$, in which the gain is much greater than the loss, the spectral width of the output varies with time according to ${ }^{9}$

$$
\Delta \nu_{\text {hom }}=\Delta \nu_{h}\left[\frac{\dot{k} t / \pi^{1 / 2} \epsilon}{1 n\left\{\left[\exp \left(k t / \pi^{1 / 2} \epsilon\right)+1\right] / 2\right\}}-1\right]^{1 / 2} \text {. }
$$

For short times $\left(k t / \pi^{1 / 2} \epsilon \ll 1\right)$ the linewidth is equal to the homogeneous width $\Delta \nu_{h}$, but for long times $\left(k t / \pi^{1 / 2} \epsilon \gg 1\right)$ Eq. (27) reduces to

$$
\Delta \nu_{\text {hom }} \simeq \Delta \nu_{h}\left[\left(\pi^{1 / 2} \in 1 n 2\right) /(k t)\right]^{1 / 2}=\Delta \nu_{h}\left\{\frac{(1 n 2)}{\left[g(0)_{\text {hom }} t\right]}\right\}^{1 / 2},
$$

where $g(0)_{\text {hom }}$ is the unsaturated line center exponential gain constant for a homogeneously broadened medium. Similarly, in an unsaturated inhomogeneously broadened amplifier the linewidth is

$$
\Delta \nu_{\text {inhom }}=\Delta \nu_{D}\left[\frac{(1 n k t-1 n 1 n\{[\exp (k t)+1] / 2\})}{(1 n 2)}\right]^{1 / 2} \text {. }
$$

For short times $(k t \ll 1)$ the linewidth is equal to the Doppler width $\Delta \nu_{D}$, but for long times $(k t \gg 1) \mathrm{Eq}$. (29) simplifies to

$$
\Delta \nu_{\text {inhom }} \simeq\left[\left(\Delta \nu_{D}\right) /(k t)^{1 / 2}\right]=\left\{\left(\Delta \nu_{D}\right) /\left[g(0)_{\text {inhom }} t\right]^{1 / 2}\right\},(30)
$$

where $g(0)_{\text {inhom }}$ is the line center unsaturated exponential gain constant for an inhomogeneously broadened medium. For both homogeneous and inhomogeneous broadening, the output spectrum becomes a Gaussian function narrowing about the center frequency of the amplifying laser transition.

For a saturated laser oscillator the results are more complicated. In the case of a homogeneously broadened medium $(\epsilon \gg 1)$, all of the factors but the Gaussian function can be removed from the integrand of Eq. (26); and the result may be written as

$$
\begin{aligned}
{\left[\partial I\left(y_{l}, t\right)\right] /(\partial t)=\left\{k /\left[\pi^{1 / 2} \in h(t)\right]\right\}\{} & {\left[I\left(y_{l}, t\right)+\eta\right] /(1} \\
& \left.\left.+y_{l}{ }^{2}\right)\right\}-\gamma I\left(y_{l}, t\right),
\end{aligned}
$$

where

$$
h(t)=\exp \left[s \int_{0}^{t .} \int_{-\infty}^{\infty} \frac{I\left(y_{n}, t^{\prime}\right)}{1+y_{n}^{2}} d y_{n} d t^{\prime}\right] .
$$

It is apparent from the form of Eq. (31) that the gain retains its Lorentzian spectral distribution in spite of saturation. In the limit of weak fields $(I \rightarrow 0)$, the function $h(t)$ is equal to unity; though after significant narrowing has occurred, the Lorentzians are effectively equal to their line center values; and Eq. (31) implies

$$
\frac{d I_{s}(t)}{d t}=\frac{k I_{s}(t)}{\pi^{1 / 2} \epsilon} \exp \left[-s \int_{0}^{t} I_{s}\left(t^{\prime}\right) d t^{r}\right]-\gamma I_{s}(t),
$$

where $I_{s}(t)=\int_{-\infty}^{\infty} I\left(y_{n}, t\right) d y_{n}$ is the total intensity within the spectral envelope. Equation (33) is in agreement with well-known relations for giant pulsing in homogeneously broadened lasers, ${ }^{10}$ and detailed numerical solutions are omitted. In the special case $\gamma \simeq 0$, Eq. (33) may be integrated analytically resulting in

$$
I_{s}(t)=I_{i} \frac{1+k /\left(\pi^{1 / 2} \in S I_{i}\right)}{1+k \exp \left\{-s I_{i} t\left[1+k /\left(\pi^{1 / 2} \in S I_{i}\right)\right]\right\} /\left(\pi^{1 / 2} \in S I_{i}\right)},
$$

where $I_{i}$ is the initial intensity. We wish to emphasize that solutions for the total intensity based on the familiar Eq. (33) are not valid until the spectrum has narrowed to a value much less than $\Delta \nu_{h}$. If such ex- 


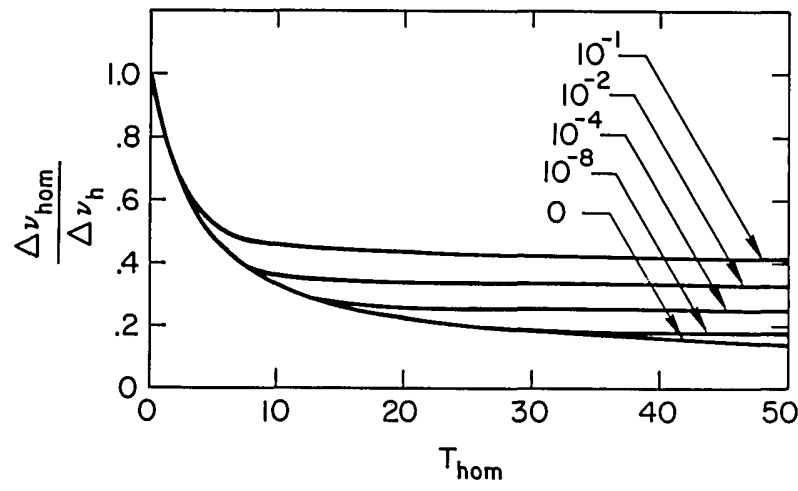

Fig. 5. Spectral narrowing in a homogeneously broadened laser for various values of $\mathrm{E}_{\mathrm{hom}}=\pi \epsilon s \eta / k$.

treme narrowing does not occur, accurate results can only be obtained directly from Eq. (31). Numerical linewidth solutions of Eq. (31) are given in Fig. 5 for the case of negligible losses. The normalized linewidth is plotted vs the dimensionless time $T_{\text {hom }}=$ $k t /\left(\pi^{1 / 2} \epsilon \ln 2\right)$ for various values of the parameter $\mathrm{E}_{\text {hom }}=\pi \epsilon s \eta / k$. Narrowing ceases when the inversion is depleted at approximately the time $T_{\text {hom }}=$ $-1 n \mathrm{E}_{\text {hom }} / 1 n 2$. At this time Eq. (28) implies that the linewidth is about $\Delta \nu_{\text {hom }}=\Delta \nu_{h}\left(-1 n 2 / 1 n \mathrm{E}_{\mathrm{hom}}\right)^{1 / 2}$, and from Eq. (34) the maximum total internal intensity is $I_{s}(t)_{\max } \simeq k /\left(\pi^{1 / 2} \epsilon s\right)$. An important conclusion is that a pulsed homogeneously broadened laser can oscillate in a large number of longitudinal modes, though single-mode behavior is expected in cw homogeneously broadened lasers.

For an inhomogeneously broadened medium $(\epsilon \ll$ 1) we assume that the intensity spectral width is large compared with $\Delta \nu_{h}$. Then the intensity distribution may be removed from the frequency integral in the saturation factor of Eq. (26) and the result simplifies to

$$
\begin{aligned}
{\left[\partial I\left(y_{l}, t\right)\right] /(\partial t),=k\left[I\left(y_{l}, t\right)+\eta\right] \exp \left(-\epsilon^{2} y_{l}{ }^{2}\right) \exp [} \\
\left.-\pi s \int_{0}^{t} I\left(y_{l}, t\right) d t\right]-\gamma I\left(y_{l}, t\right) .
\end{aligned}
$$

This equation has the same form as Eq. (33), and the usual giant pulse solutions apply to each spectral region. In Fig. 6 are numerical linewidth solutions for the case of negligible losses. In this figure the normalized linewidth is plotted against the time $T_{\text {inhom }}$ $=k t$ for various values of $\mathrm{E}_{\text {inhom }}=\pi s \eta / k$. It is evident from the figure that saturation leads to a rebroadening of the intensity distribution back to the inhomogeneous (Doppler) line shape. The minimum linewidth occurs at the time $T_{\text {inhom }} \simeq-1 n \mathrm{E}_{\text {inhom }}$, when the width is about $\Delta \nu=\Delta \nu_{D}\left(-1 n \mathrm{E}_{\text {inhom }}\right)^{-1 / 2}$.

\section{Applications}

The results derived here can be applied directly to obtain information about the parameters of practical laser systems. As an example, we consider the 1700$\AA$ xenon laser transition and assume that the broad- ening is primarily homogeneous. ${ }^{11,12}$ Data for the xenon laser show an exponential gain constant of about $g(0)_{\text {hom }} \simeq 4 \times 10^{8} \mathrm{sec}^{-1}$ and a pulse delay of about $t \simeq 5 \times 10^{-8}$ sec. (See Ref. 12.) The expected spectral narrowing by the factor $\left[g(0)_{\text {hom }} t / 1 n 2\right]^{-1 / 2}$ $=5.4$ is in fair agreement with the reported narrowing from $200 \AA$ to $30 \AA$. Also, from the depletion relation $g(0)_{\text {hom }} t=-1 n \mathrm{E}_{\text {hom }}$ (or Fig. 5), it follows that the parameter $\mathrm{E}_{\mathrm{hom}}$ has the approximate value 2 $\times 10^{-9}$. The saturation parameter $s$ could be readily inferred from the peak output intensity if the coupling were known. These parameters may then be used to obtain information about the fundamental coefficients and densities of the laser medium.

Alternatively, if the parameters of the laser medium are known, this formalism can be used to obtain quantitative estimates of the output power, number of modes, etc. in conventional pulsed lasers. We illustrate this procedure by using typical numbers for a $6943 \AA$ pulsed ruby laser. Ruby at room temperature is predominantly homogeneously broadened and the parameter $\mathrm{E}_{\text {hom }}=\pi \epsilon s \eta / k=\pi^{1 / 2} s \eta / g(0)_{\text {hom }}$ must be estimated first. For simplicity we use typical experimental values rather than first principle calculations. It is assumed that the ruby rod has a cross section of $1 \mathrm{~cm}^{2}$, a length of $10 \mathrm{~cm}$, and is positioned in a cavity of 1-m length. In such a laser, the unsaturated intensity approximately doubles each round trip, so the gain is $g(0)_{\mathrm{hom}}=(c / 2 L) 1 n 2=1.04 \times 10^{8}$ $\mathrm{sec}^{-1}$. For a saturation energy of $1 \mathrm{~J}$, one finds $s=1$ $\mathrm{cm}^{2} / \mathrm{J}=10^{-4} \mathrm{~m}^{2} / \mathrm{J}$. At room temperature the linewidth of the $6943 \AA$ transition is $\Delta \nu_{h}, 11 \mathrm{~cm}^{-1}=3.3$ $\times 10^{11} \mathrm{~Hz}$, so the spontaneous emission noise parameter is $\eta=h \nu_{l} \Delta \nu_{h} / 2 A=4.72 \times 10^{-4} \mathrm{~J} / \mathrm{m}^{2} \mathrm{sec}$. (See Ref. 13). Combining these results, one obtains $\mathrm{E}_{\text {hom }}$ $=8.05 \times 10^{-16}$ and the output spectrum has a minimum linewidth of $\Delta \nu_{\text {hom }}=\Delta \nu_{h}\left(-1 n 2 / 1 n \mathrm{E}_{\mathrm{hom}}\right)^{1 / 2}=$ $1.55 \mathrm{~cm}^{-1}=4.66 \times 10^{10} \mathrm{~Hz}$. This result is rather insensitive to the value of $E_{h o m}$, and a factor of ten change in $s, \eta$, or $g(0)_{\text {hom }}$ causes only a $3 \%$ change in $\Delta \nu_{\text {hom. }}$. Fair agreement with experiment is obtained, and it has been found that the actual emission linewidth of a pulsed room temperature ruby laser operating far above threshold is about $1.2 \mathrm{~cm}^{-1}$ insensitive to the pumping level. ${ }^{14}$

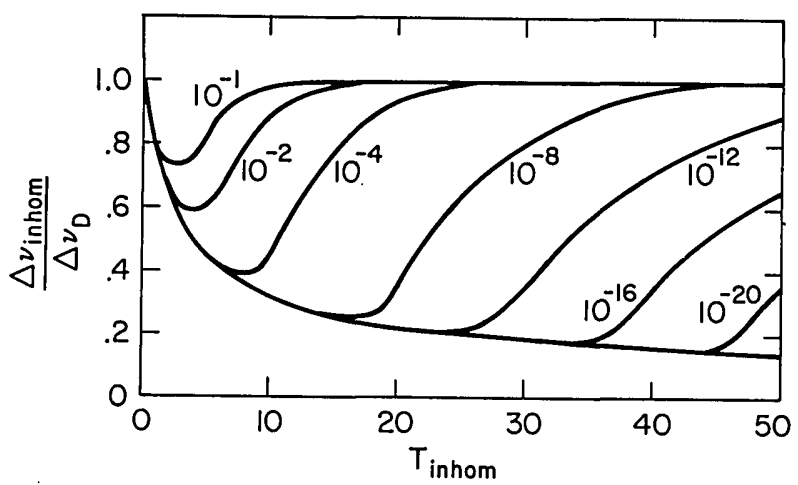

Fig. 6. Spectral narrowing in an inhomogeneously broadened laser for various values of $E_{i n h o m}=\pi s \eta / k$ 
Since the theoretical and experimental emission linewidths are large compared with the mode spacing, multimode behavior is obtained. For a linewidth of $1.5 \mathrm{~cm}^{-1}$ and a mode spacing of $\Delta \nu=c / 2 L=$ $1.5 \times 10^{8} \mathrm{~Hz}$, one obtains about 300 modes. This is in contrast to the behavior of steady-state, homogeneously broadened lasers where single-mode oscillation is usually expected as the mode nearest line center clamps the gain at its threshold value. In a pulsed laser the initial gain may greatly exceed the loss for a large number of modes; and spatial holeburning is not required to account for the multimode effects.

With the results of Sec. II, the oscillation linewidth of the longitudinal modes may also be readily calculated. For the instantaneous switching model, the linewidth is given by Eq. (20). If the initial round trip gain at the frequency of one of the modes of the ruby laser is a factor of two $(F=1.414)$ and the mode spacing is $\Delta \nu=1.5 \times 10^{8} \mathrm{~Hz}$, it follows that the linewidth is $\Delta \nu_{\text {mode }}=\Delta \nu(F-1) / \pi F^{1 / 2}=16.6 \mathrm{MHz}$. Thus, in a high gain pulsed laser, the oscillation linewidth may be much greater than in conventional $\mathrm{cw}$ lasers. Linewidth considerations are essential for applications such as pulsed holography, where a large coherence length is required.

\section{Conclusion}

In summary, the techniques described here make possible a quantitative discussion of the spectral properties of pulsed lasers. It has been shown that narrow mode resonances develop after a few round trips of the radiation initiated by spontaneous emission, provided the gain is not too large. For very high gain systems $(F \geq 10)$, the resonance structure does not occur. Also, the over-all spectral envelope narrows about line center. An understanding of these effects is important for the prediction of a pulsed laser's output characteristics and for a diagnosis of the fundamental parameters of a laser medium. The results make possible a quantitative discussion of uv and x-ray systems where the high levels of spontaneous emission make data interpretation difficult. In such systems even the decision as to whether lasing has been achieved is sometimes not straightforward.

This work was supported in part by the National Science Foundation.

\section{References}

1. M. Menat, J. Appl. Phys. 36, 73 (1965), and the references.

2. I. S. Gradshteyn and I. W. Ryzhik, Table of Integrals, Series, and Products (Academic Press, New York, 1965), Sec. 3.61.

3. H. Kogelnik and A. Yariv, Proc. IEEE 52, 165 (1964).

4. E. I. Gordon, Bell Syst. Tech. J. 43, 507 (1964).

5. A. L. Schawlow and C. H. Townes, Phys. Rev. 112, 1940 (1958).

6. A. Yariv, Quantum Electronics (Wiley, New York, 1967), Chap. 24.

7. K. R. Manes and A. E. Siegman, Phys. Rev. A 4, 373 (1971).

8. E. I. Gordon, A. D. White, and J. D. Rigdon, in Optical Masers, J. Fox, Ed. (Polytechnic Press, Brooklyn, 1963), p. 309.

9. L. W. Casperson and A. Yariv, IEEE J. Quantum Electron. QE-8, 80 (1972).

10. A. Yariv, Introduction to Optical Electronics, (Holt, Rinehart and Winston, New York, 1971), Eqs. (6.7-2) and (6.7-3).

11. H. A. Koehler, L. J. Ferderber, D. L. Redhead, and P. J. Ebert, Appl. Phys. Lett. 21, 198 (1972).

12. J. B. Gerardo and A. W. Johnson, IEEE J. Quantum Electron. QE-9, 748 (1973).

13. A. L. Schawlow, Advances in Quantum Electronics, J. R. Singer, Ed. (Columbia U. P., New York, 1961), p. 53.

14. C. L. Tang, H. Statz, and G. De Mars, J. Appl. Phys. 34, 2289 (1963).

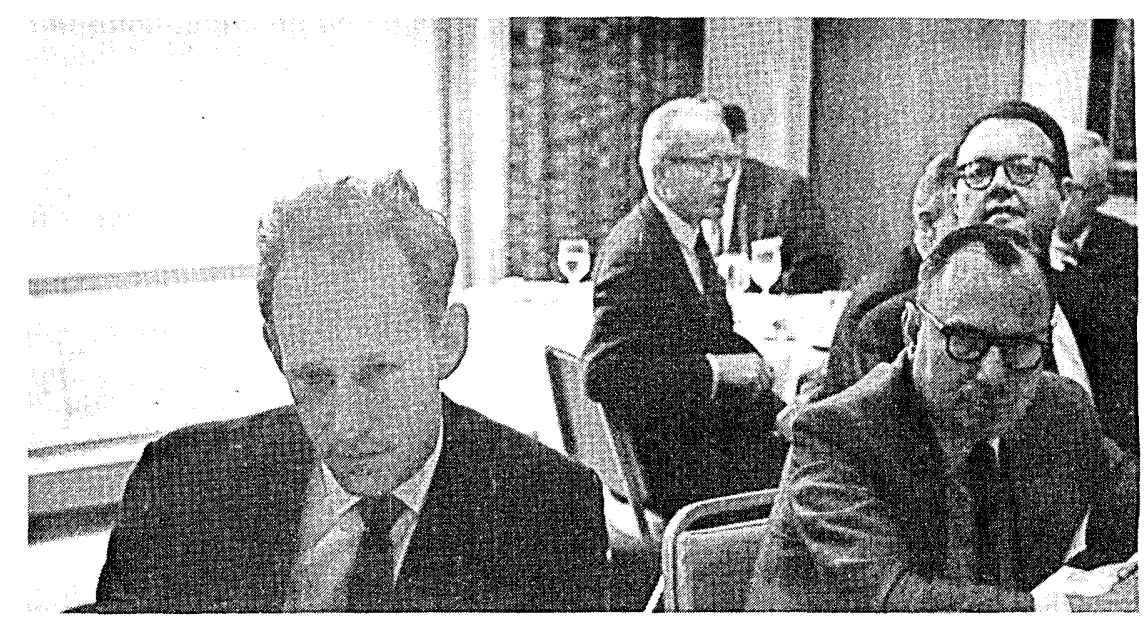

S. F. Jacobs of the University of Arizona, F. S. Harris, Jr., of Old Dominion University, A. J. Cussen of Electro-Optical Industries, and Eric Wormser. Photo: D. MacAdam, Eastman Kodak. 